\title{
Millennium Development Goals(MDG 4 andMDG 5 and Child and Maternal Health Status in Bangladesh
}

(Birdem Med J 2014; 4(2):66-68.)

Bangladesh has made commendable progress in achieving Millennium Development Goals (MDGs) 4 and 5 in the last decade ${ }^{1}$. The vision of the set target is not only to achieve the MDGs 4 and 5 but also to reduce child mortality and improve maternal health, with women and children enabled to realize their right to the highest attainable standard of health by the year 2015 and beyond.

MDG4 has 3 components - goal, target and indicator. Goal 4 is to reduce child mortality with a target (4A) to reduce under- 5mortality by two-thirds, between 1990 and 2015; It has 3 indicators - Indicator 4.1: Reduce underfive mortality rate (48/1000 live births), Indicator 4.2: Reduce infant mortality rate (31/1000 live births) and Indicator 4.3: Increase proportion of 1 year-old children immunized against measles $(100 \%)$ ) by the year $2015^{2}$.

We have considerable improvement in child survival, placing us among only 16 countries in the world that are on track to achieve MDG-4 with regard to child mortality ${ }^{3}$. Trend shows significant reduction in under -5 , infant and neonatal mortality since mid 90s. Proportion of neonatal, infant and under five deaths in Bangladesh was - children 1-5 yrs (19\%), infant (21\%), newborn 60 $\%$ respectively. ${ }^{4}$

A number of child survival interventions has been adopted to reduce deaths and to improve child health and development. Delivery of these interventions led to impressive decline in infant and child deaths but neonatal death rates remain unacceptably high which is one of the major challenges in achieving MDG 4 . There are 3 major causes that account for $88 \%$ of all newborn deaths (Intrapartum related complications, severe infection and preterm complications $)^{5} .60 \%$ of all child deaths are due to-birth asphyxia, preterm baby, pneumonia and other serious infections. Malnutrition is a great factor.Globally more than one third of children deaths are due to malnutrition. In Bangladesh - $43 \%$ of children are stunted, $16 \%$ severely stunted, $17 \%$ of under-5 children wasted and 3\% severely wasted. 4

There is considerable lacking in the essential newborn care practice such as immediate drying (6\%), wrapping within 5 minutes ( $2 \%$ ) and delayed bathing of the baby until after first 72 hours (less than 20\%). Only 22.6\% newborns receive postnatal care by a medically trained provider within 48 hours of birth and $9.5 \%$ receive care from non-medically trained providers. 6

Integrated Management of Childhood Illness (IMCI): It is a public health strategy for the preventive and curative care of sick children to set of evidence-based clinical guidelines, for the outpatient care of sick children and first-level referral care of sick children. It can be provided at home, first level health care facility, and specialized hospital. So it is to improve workers skills, improve the health systems, improve family \& community practices (WHO 2012). In 2011 about 71\% with suspected pneumonia had received appropriate antibiotic in comparison to $21 \%$ in 2001. One in four children with diarrhea was taken to health facility and $78 \%$ were given ORS. $^{4}$

Infant and Young Child Feeding (IYCF): Specific Objectives of National IYCF Strategy :different organizations have confirmed increased breast feeding practice among mothers(initiation at first hour, exclusive at 6 months and maintenance from 6-9 months)

EPI(expanded programme of immunization) :Our most successful public health intervention programme. The current routine immunization programme includes tuberculosis, diphtheria, pertusis, tetanus, hepatitis-b, hemophilus influenza $b$, poliomyelitis, measles and rubella vaccine. In Bangladesh there is universal access to immunization and 1.2 million deaths have been prevented from 1987-2000. Immunization programme in 2011 could cover $83 \%$ of all children at one year of age 
fully vaccinated which was 51\% in 1991 and $53 \%$ in the year 2000.(source CES) .Current record shows 60\% children (6-55 months) received Vit-A supplementation, $50 \%$ children under 5 yrs have received deworming medication. Bangladesh already has been declared polio free country.

Although the above progress puts Bangladesh on track for reaching the MDG-4 target by 2015 but reduction in neonatal mortality remain a significant issue..1.5 million (38\% of all newborn) deaths occur in 4 countries of South Asia. 99\% of newborn deaths are in low/middle income countries- $66 \%$ in Africa and South Asia. Approx $67 \%$ of global total neonatal deaths are occurring in 10 countries of Asia and Africa We have 3.1 Million newborn deaths in 2010 with $28 \%$ decline in 2 decades ranking 7 where India rank 1 and Pakistan being $3 \mathrm{rd}$. For improvement of neonatal health, needs continuum of care i.e. maternal health \& nutrition, antenatal check up, safe delivery and postnatal care.

There are certain key strategies to achieve MDG 4:

1. Strengthen the existing routine EPI - continue polio eradication, emphasize on elimination of neonatal tetanus measles and scale up new vaccination.

2. Scale up the implementation of IMCI strategies.

3. Continue the existing acute respiratory infection, diarrheal diseases and malaria control programme in areas which currently don't have IMCI.

MDG 5(Goal 5): Is to improve maternal health by 2015 with a target( target $5 \mathrm{~A}$ ) to reduce maternal mortality by three-quarters, between 1990 and 2015. It has six indicators.Indicator 5.1 Maternal mortality ratio,indicator 5.2 proportion of births attended by skilled health personnel, indicator 5.3 contraceptive prevalence rate ,indicator 5.4 Adolescent birth rate, and indicator 5.5 antenatal care coverage (at least one visit and at least four visits), Indicator 5.6 Unmet need for family planning. Fifth mellinium devolopment goal(MDG 5) established the goal of a $75 \%$ reduction in the maternal mortality ratio(MMR -number of maternal death per 100000 livebirth).between 1990 to 2015 .. . To be classified as maternal,pregnancy need to be a causal factor in death.It can be either have a direct effect(complication of pregnancy or child birth or postpartum complications)or indirect effect(exacerbation of a preexisting condition). There for accident or incident which have pregnancy has no role is not classified as maternal death.All definition include direct and indirect causes during pregnancy and within 6 weeks of the termination of pregnancy.

.MDG monitoring(2012)mentioned that every year in developing countries 500000 women die during the child delivery.In Bangladesh 2005 in 570 women died out of 100000 during child delivery.Between 1996 and 1997,26\% maternal death occurred in Banggladesh due to unsafe abortion practices.. USAID-Bangladesh $(2011)^{6}$ stated that $40 \%$ maternal death declined in nine years from 2001 to 2010 to 194 maternal death per 100000 live birth .However,MDG's aim is to be at 144 per 100000 life birth by 2015. The rate of decline was at an averae of $5.5 \%$ per year,compared to the average annual rate reduction of 5.45 required for achieving the mellinium development goal 5. Maternal mortality declined in almost all ages in two surveys. The entire decline in maternal mortality was due to reductions in direct obstratic deaths. Maternal mortality during preanancy and delivery declined by $50 \%$ and postpartum maternal death decreased by $34 \%$. The dominant causes of direct obstratic deaths were hemorrhage $(31 \%)$, eclampsia (20\%),odstructed or prolonged labour(7\%) and abortions(1\%).Between 2001 and 2010 the maternal mortality ratio due to hemorrhage decrease by $35 \%$ to eclampsia by $50 \%$ and to obstructed labour by $26 \%{ }^{7}$. The second Bangladesh national maternal moratlity and health care survey was conducted in 2010.Bangladesh appears to be in the track to achieve Mellinium goal by 2015 but need to make continued improvement to achieve it.

The following challenges will need to receive special attention if Bangladesh wishes to achieve MDGs $4 \& 5$.

1. High proportion of home delivery.

2. High prevalence of malnutrition.

3. Lack of skilled birth attendants and trained human resources.

4. Low coverage and quality of antenatal, post-natal and sick newborn care.

5. Poor care seeking behavior

6. Deep-seated community norms and practices.

7. Lack of access to information, care and social support.

Acknowledgement: Child health status and achieving MD4 was an excerpt from the "Prof. SGM CHOWDHURY 
memorial oration at $25^{\text {th }}$ Annual scientific cinference of Association of Physiscians of Bangladesh delivered by Prof.Nazmun Nahar, Professor of Pediatrics and past president of Bangladesh college of Physicians and Surgeons.

\section{References:}

1. Chowdhury S, Banu LA, Chowdhury TA, Rubayet S, Khatoon S. Achieving Millennium Development Goals 4 and 5 in Bangladesh. BJOG 2011; 118 (suppl 2): 36-46.

2. United Nations Development Project (UNDP) report 2013. Bangladesh status.

3. Countdown to 2015 Initiatives. Countdown to 2015 Maternal Newborn \& Child survival: Tracking Progress in
Maternal, Newborn \& Child Survival; the 2008 report. Geneva: UNICEF, 2010[www.countdown2015mnch.org]

4. National Institute of Population Research and Training, Mitra and Associates and Macro International. Bangladesh demographic and health survey, 2011. Dhaka, Bangladesh: NIPORT, 2011.

5. Lawn JE et al BJOG Sept 2009. Data sources: Estimates of neonatal (2008) deaths from WHO. Updated June 2010.

6. National Institute of Population Research and Training, USAID, AusAID, Measure evaluation, UNFPA and ICDDRB. Bangladesh Maternal Mortality and Health Care Survey (BMMS) 2010, Summary of key findings and Implications. Dhaka, Bangladesh: Bureau of Statics, 2010.

7. 7.ICDDR,B.Health and science Bulletin. June 2011;9 ( 2): 1,2 . 